\title{
Interactive comment on "The physics of fault friction: Insights from experiments on simulated gouges at low shearing velocities" by Berend A. Verberne et al.
}

\section{Anonymous Referee \#2}

Received and published: 12 August 2020

General comments: This paper summarizes experimental, microstructural, microphysical, and numerical modeling studies of the frictional behavior of simulated gouge conducted at Utrecht University (UU) in the past two or so decades. Although the paper does not have any new results, except some of the modeling results only presented in the recent international conference, it includes the basic information on the experimental setup and results, microphysical model, and numerical modeling of earthquake cycles using their friction model (CNS model). The paper is based on many previous papers presented by UU group and hence it is very well written. Therefore, I recommend publication only after the following minor comments shown below. 
Specific comments:

Quality of figures: As mentioned in the reviewer 1, overall quality of figures is too low. Some of the words can't be read. Hence it should be improved.

The difference in the frictional properties. Line 258-inset of Fig. 5b: I assume that the authors try to mention the similarity of temperature dependences between calcite (Fig. 5) and qtz-phyllosilicate mixture (Fig. 4a) gouges. However, their variations as a function of temperature are different. In particular, calcite gouges show a wide region of negative $v$ dependence with sharp peaks at $500 \mathrm{C}$. Can you elaborate more on the difference? Because as shown in later, the CNS model offers fault friction law based on microphysics supported by those experiments. If the authors can illuminate tho difference in terms of rock and mineral physics aspects, this will give a more generalized view on the microphysics of fault friction. I assume that is a point the CNS model aims.

Robustness of the CNS model As repeatedly mentioned in the paper, CNS model is based on microphysics supported by experimental results. In that sense, the CNS model provides a transparent origin of the constitutive parameters. However, as shown in Fig. 9 and Chapter 7 (Remaining challenges), the CNS model has a significant shortcoming on that it can only reproduce slow slip or earthquakes with limited coseismic displacement. Hence, I guess that the authors should avoid bold statements on the robustness of the model (e.g., lines $25,361,468$, and so on).

Lines 169-170: "The maximum rotation or shear displacement that can be achieved is limited by the water cooling and pore fluid systems, ". What does that mean? It is better to elaborate more on the experimental detail for readers outside of the field.

Line 240 and Fig. 4: Need a more detailed explanation of the Fig. 4 (in particular 4c). For example, explanations on the differences in color and meaning of peaks in dash-lines are needed.

Line 250: "an important role for the presence of (pressurized) pore water (Fig. 5a- 
insets) "But how can we understand the importance of ( (pressurized) pore water) from Fig. 5a?

Interactive comment on Solid Earth Discuss., https://doi.org/10.5194/se-2020-85, 2020.

Interactive

comment 